\title{
Atrial fibrillation II: Rationale for surgical treatment
}

\author{
James L. Cox, MD
}

See related editorial on page 1686.
From the Division of Cardiothoracic Surgery, Washington University School of Medicine, St Louis, Mo.

Received for publication May 31, 2003; accepted for publication June 9, 2003.

Address for reprints: James L. Cox, MD, 13523 Rosewood Ln, Naples, FL 34119 (E-mail: jamescoxmd@aol.com).

J Thorac Cardiovasc Surg 2003;126:1693-9

Copyright (C) 2003 by The American Association for Thoracic Surgery

$0022-5223 / 2003 \$ 30.00+0$

doi:10.1016/j.jtcvs.2003.06.003

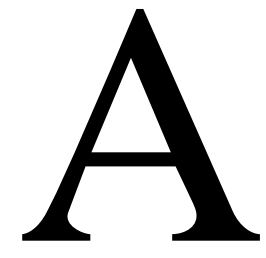

11 surgical procedures for cardiac arrhythmias can be classified as either isolation procedures or ablation procedures. Isolation procedures do not actually terminate arrhythmias but rather confine them, their trigger mechanisms, or both to a desired region of the heart to minimize their adverse effects. Examples of isolation procedures include the following:

1. elective His bundle ablation for any type of supraventricular tachycardia, which confines the supraventricular tachycardia to the atria;

2. the left atrial isolation procedure for automatic left atrial tachycardias and atrial fibrillation, which confines the tachycardia or fibrillation to the left atrium;

3. the right atrial isolation procedure for automatic right atrial tachycardias, which confines the tachycardia to the right atrium;

4. the corridor procedure for atrial fibrillation, which confines the fibrillation to the atria and isolates it from the atrioventricular node;

5. the right ventricular isolation procedure for nonischemic ventricular tachycardia, which confines the tachycardia to the right ventricle; and

6. pulmonary vein isolation for intermittent atrial fibrillation, which confines the trigger for atrial fibrillation to the pulmonary veins.

Ablation procedures preclude arrhythmias from developing either by destroying their trigger mechanism or by altering (or removing) the substrate that allows the arrhythmia to be induced and maintained. Ablative procedures include the following:

1. surgical intervention for the Wolff-Parkinson-White syndrome, which interrupts the macroreentrant circuit responsible for the tachycardia;

2. discrete cryosurgery for atrioventricular node reentry tachycardia, which interrupts the microreentrant circuit responsible for the tachycardia;

3. focal cryoablation for automatic atrial tachycardias, which destroys the trigger mechanism responsible for the tachycardia;

4. endocardial resection procedures for ischemic ventricular tachycardia, which removes the microreentrant circuit responsible for the tachycardia;

5. endocardial cryosurgical procedures for ischemic ventricular tachycardia, which destroys the microreentrant circuit responsible for the tachycardia; and

6. the maze procedure for atrial fibrillation, which destroys the macroreentrant circuits responsible for atrial fibrillation.

The ultimate effect of any cardiac surgical procedure on its target patient group depends on its safety and efficacy. From a practical standpoint, however, the complexity of the operation might be more important in determining whether it is adopted by enough surgeons to have a significant effect on its subject population. Thus it is clear that if we are to develop a surgical procedure that will be applicable to the masses of patients with atrial fibrillation, 3 interacting factors of the procedure must be addressed: complexity, adoptability, and efficacy.

The complexity and adoptability of a surgical procedure are inversely related to one another, and the efficacy of the procedure, in this case its ability to cure atrial fibrillation, is independent of those 2 factors (Figure 1). By using this model, perhaps the nearest thing to a perfect cardiac surgical procedure is a pacemaker implantation for heart block (Figure 2); it is virtually always curative (efficacy = $100 \%$ ) and is extremely simple to perform (complexity $=0$ ), and therefore the 


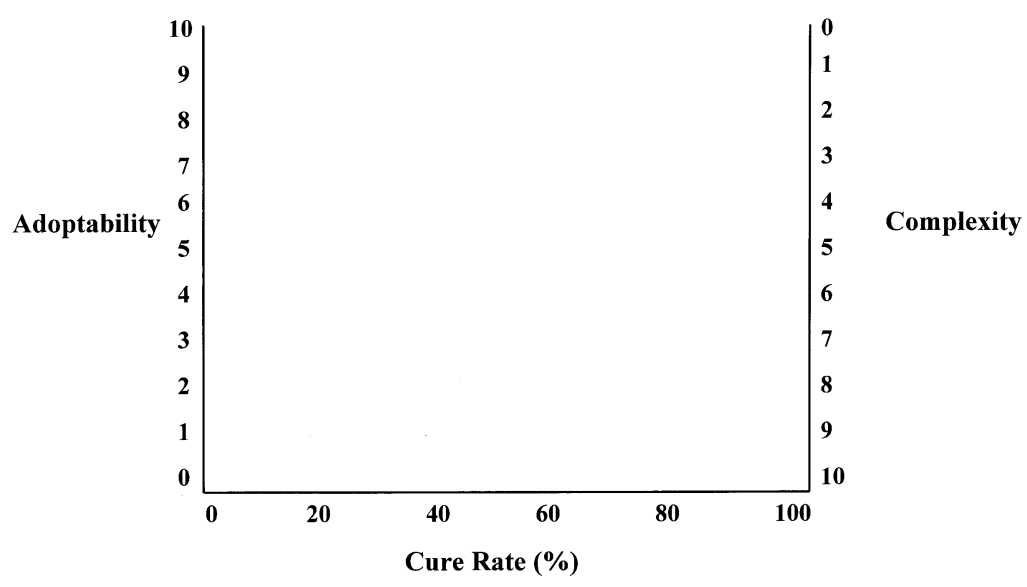

Figure 1. The effect of a given surgical procedure depends not only on its efficacy in curing the surgical problem but also on its complexity, which is inversely related to its adoptability.

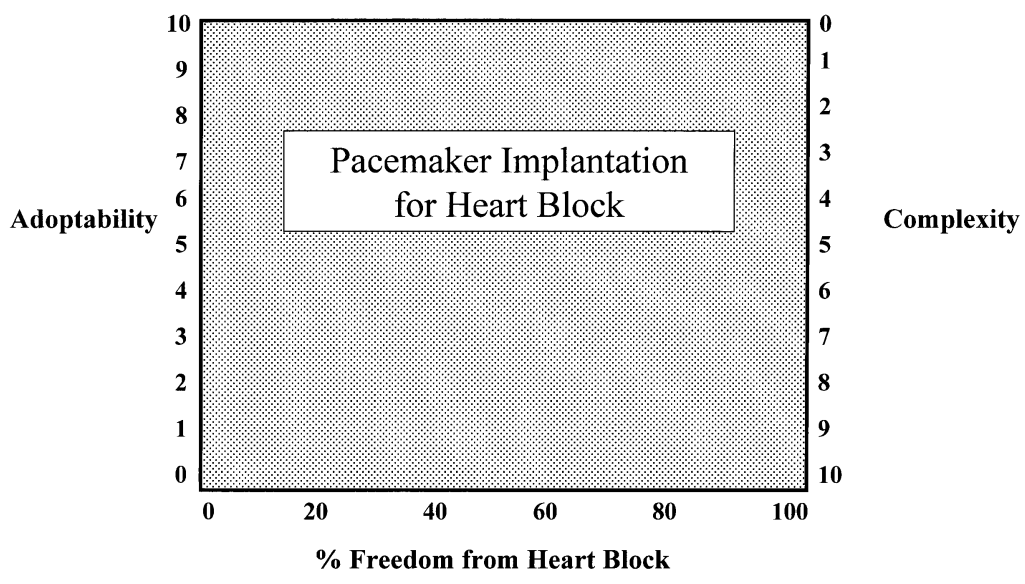

Figure 2. The ideal surgical procedure is $100 \%$ curative and is so simple technically that all surgeons will adopt it as therapy. Perhaps the best example of such an ideal operation is pacemaker implantation for heart block.

surgeon-cardiology team uses this form of treatment for all cases of heart block (adoptability $=10$ ).

These relationships have been particularly important in the surgical treatment of atrial fibrillation because the maze procedure is technically challenging, and despite its effectiveness in curing atrial fibrillation, it has been used by relatively few surgeons since it was first performed clinically in 1987. For example, an assessment of the effect of the surgical maze III procedure using the model described above produces dramatically different results (Figure 3). The maze III procedure is so complex (eg, 9.5 of 10) that its adoptability is minimal ( 0.5 of 10$)$. This results in an operation that despite being safe (operative mortality $=<1 \%$ ) and effective (cure rate $=>98 \%$ ) has had little direct effect on the absolute number of patients cured of atrial fibrillation.

Similar evaluations can be made for the other less complex operations that are currently being used for the surgical treatment of atrial fibrillation. The most common of these procedures is simple pulmonary vein isolation. Both surgeons and interventional cardiologists have favored using radiofrequency, microwave, cryothermia, or laser energy sources over the old cut-and-sew technique for creating lines of conduction block, whether they are performing simple pulmonary vein isolation or placing additional lesions in the left, right, or both atriums. Overall, pulmonary vein isolation with these energy sources has resulted in an initial cure rate of approximately $60 \%$ for atrial fibrillation (efficacy $=60 \%$ ). Despite this procedure being less effective for atrial fibrillation than the maze III procedure, it is much simpler to perform (complexity $=3$ of 10 ), and therefore its adoptability is greater ( 7 of 10). The result is that the absolute number of patients who have been cured of atrial fibrillation with this simpler but less effective procedure dwarfs the number cured with the maze III procedure (Figure 4). This observation documents the importance of a 


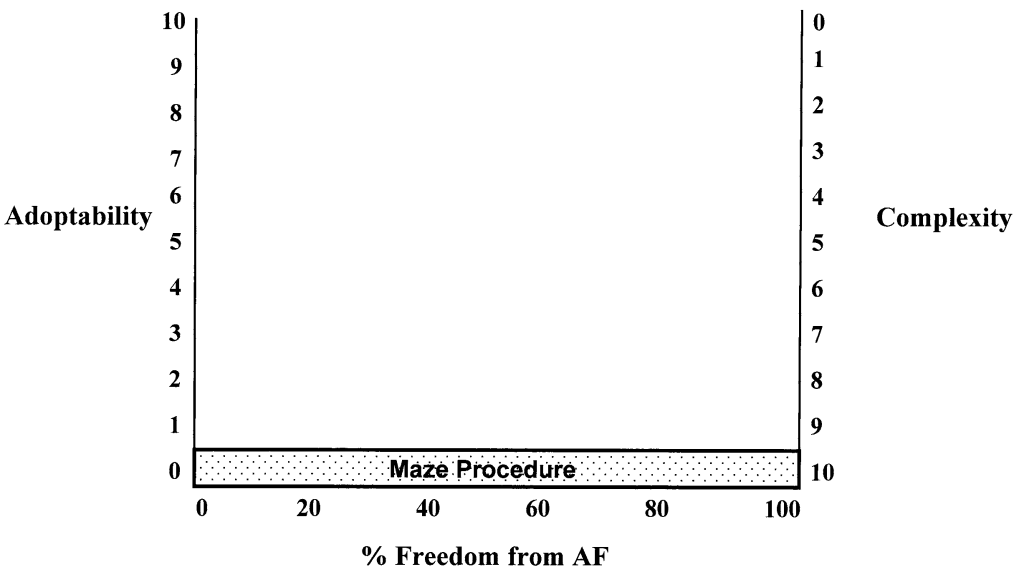

Figure 3. When performed correctly, the surgical maze procedure cures essentially all patients with atrial fibrillation. However, it is such a complex procedure that very few surgeons have adopted it, and as a result, the absolute number of persons with atrial fibrillation who have benefited directly from the maze procedure has been minimal. AF, Atrial fibrillation.

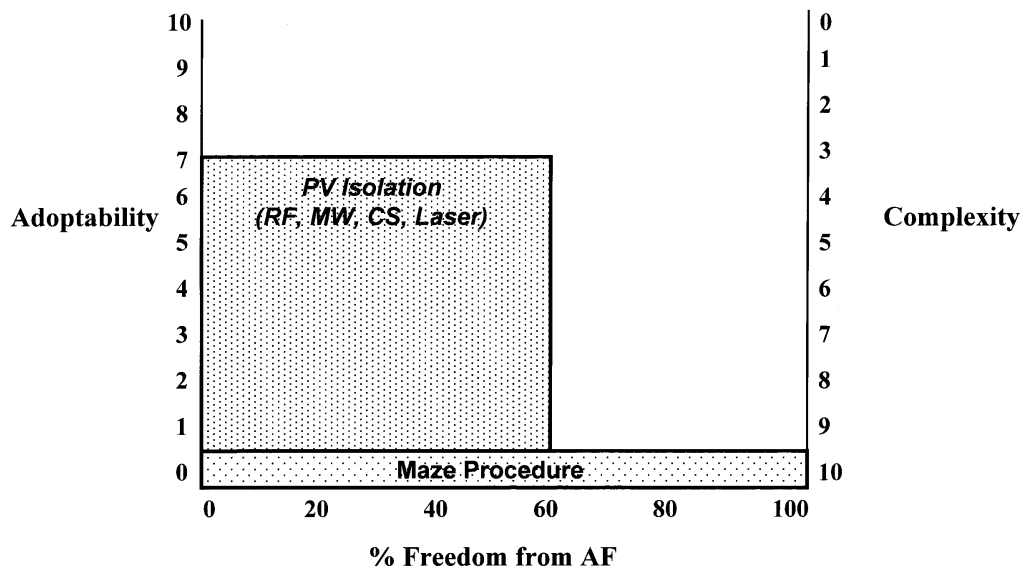

Figure 4. Simple pulmonary vein isolation performed with currently available energy sources is much less effective than the maze procedure in curing atrial fibrillation. However, its technical simplicity has resulted in its being adopted by many surgeons and interventional cardiologists, and as a result, its effect on the treatment of atrial fibrillation has been greater than that of the more effective maze procedure. $R F$, Radiofrequency; $M W$, microwave; $C S$, cryosurgery; $A F$, atrial fibrillation.

procedure's adoptability in comparison with its effectiveness in present clinical practice.

When an ablative strategy is added to the isolation strategy of simple pulmonary vein isolation (Figure 5), the cure rate for the procedure increases as expected (efficacy $=$ $90 \%$ ), but the procedure becomes more difficult to perform (complexity $=7$ of 10), and therefore the adoptability decreases ( 3 of 10). Thus although the cure rate increases with these additional ablative lesions, the absolute number of patients cured decreases because not as many surgical teams will perform the more complex procedure.

These frustrating practical relationships involving the complexity, adoptability, and efficacy of atrial fibrillation procedures have now reached an apparent impasse that has stifled the progress that was made earlier in this field. Fortunately, however, several important observations have resulted from the clinical experience accumulated by both cardiologists and surgeons over the past few years that make the future possibilities quite exciting.

We have learned empirically, for example, that many of the lesions of the original surgical maze III procedure might not be absolutely essential for the ablation of atrial fibrillation. The maze procedure was designed to preclude the development of macroreentry anywhere and everywhere in the atria. There is emerging evidence, however, that although continuous atrial fibrillation is maintained by mul- 


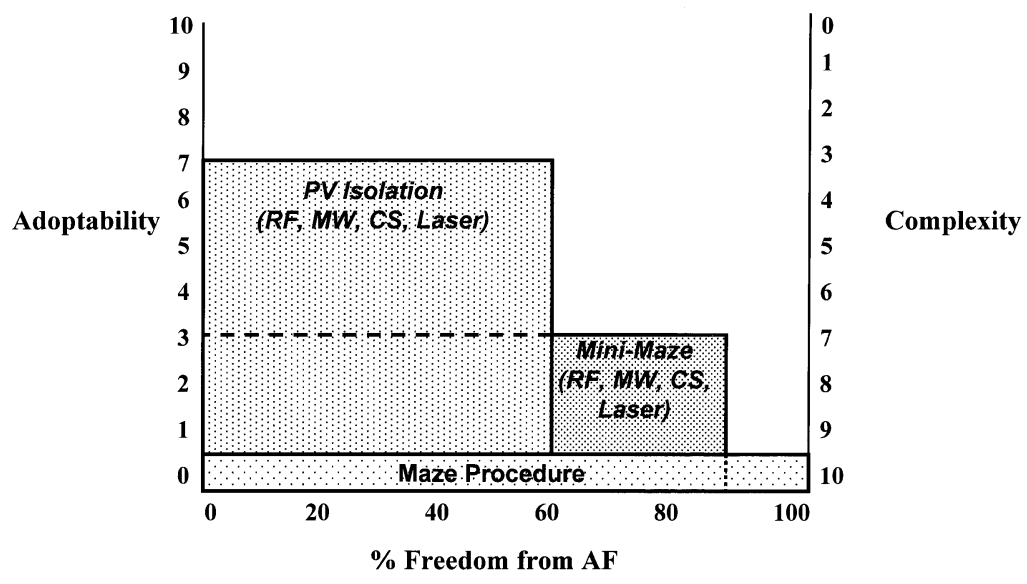

Figure 5. The addition of one or more linear lesions to simple pulmonary vein isolation results in a significantly higher cure rate of atrial fibrillation. However, the added complexity of the procedure in comparison with simple pulmonary vein isolation decreases its adoption by surgeons and cardiologists, resulting in an overall decrease in the number of patients with atrial fibrillation who benefit from the procedure. $R F$, Radiofrequency; $M W$, microwave; $C S$, cryosurgery; $A F$, atrial fibrillation.

tiple macroreentrant circuits, there might be only a limited number of sites in the left atrium that are capable of sustaining such circuits. ${ }^{1}$ In that case it would not be necessary to place lesions in the atrium that are capable of precluding all theoretic macroreentrant circuits (as in the maze procedure) because in reality, those circuits might always appear in only a few specific sites. This is the theory underlying the so-called focal atrial fibrillation that seems to respond to critically placed lesions away from the pulmonary veins. It also supports the empiric observation that many of the lesions in the maze procedure can be eliminated and yet still cure continuous atrial fibrillation (Figure 6).

For example, we have never been convinced that the septal lesion was absolutely essential to the efficacy of the maze procedure and performed it primarily to gain better exposure of the inside of the left atrium. Recent interventional and surgical therapy for atrial fibrillation would suggest that the septal lesion might not be necessary. In addition, the lesion extending out into the left atrial appendage was made a part of the maze procedure to preclude the theoretic possibility of reentry occurring around the base of the appendage. That reentrant circuit has never been documented and might well not occur clinically. Therefore that lesion might also be unnecessary.

If neither the septal nor the left atrial appendage lesions are critical to the ablation of atrial fibrillation, the essential left atrial lesions would be reduced to only the pulmonary vein encircling incision and the lesion across the isthmus between the inferior pulmonary veins and the mitral annulus. Experience with the maze procedure, as well as with other surgical arrhythmia procedures, has repeatedly confirmed that the left atrial isthmus lesion is extremely impor- tant in abolishing the reentry responsible for atrial fibrillation. Indeed, this lesion, along with its companion cryolesion in the coronary sinus, has proved to be the Achilles heel of the maze procedure in that every failure in our own series was shown to have conduction across this isthmus postoperatively. ${ }^{2}$

In addition to the possibility of streamlining the left atrial lesions, the experimental observations of Lammers and colleagues ${ }^{3}$ regarding the importance of refractory period differentials in the left and right atrium suggested that the right atrial lesions of the maze procedure might be replaced by a simpler approach. Their observations have now been confirmed clinically. It is well established that the duration of the local refractory period determines the minimum size that a macroreentrant circuit can be at that site in the atrium. ${ }^{4}$ Because the refractory periods are shorter in the left atrium, it can sustain the relatively smaller macroreentrant circuits that are characteristic of atrial fibrillation. Because the refractory periods are relatively longer in the right atrium, it is probably not capable of sustaining atrial fibrillation by itself unless it is pathologically enlarged. Thus the treatment of atrial fibrillation can be focused on the left atrium with the knowledge that if macroreentry can be prevented by certain critical lesions placed there, atrial fibrillation will not recur. The right atrium, which is capable of sustaining only atrial flutter, can then be addressed by placing a lesion in the isthmus between the coronary sinus and the tricuspid valve, a critical limb of the macroreentrant circuit responsible for the vast majority of clinical atrial flutter. ${ }^{5}$ In summary, it would appear that by placing the following lesions, most patients with atrial fibrillation of either type could be cured: pulmonary vein encircling incision, left atrial isthmus lesion with its attendant coronary sinus lesion, and right atrial 


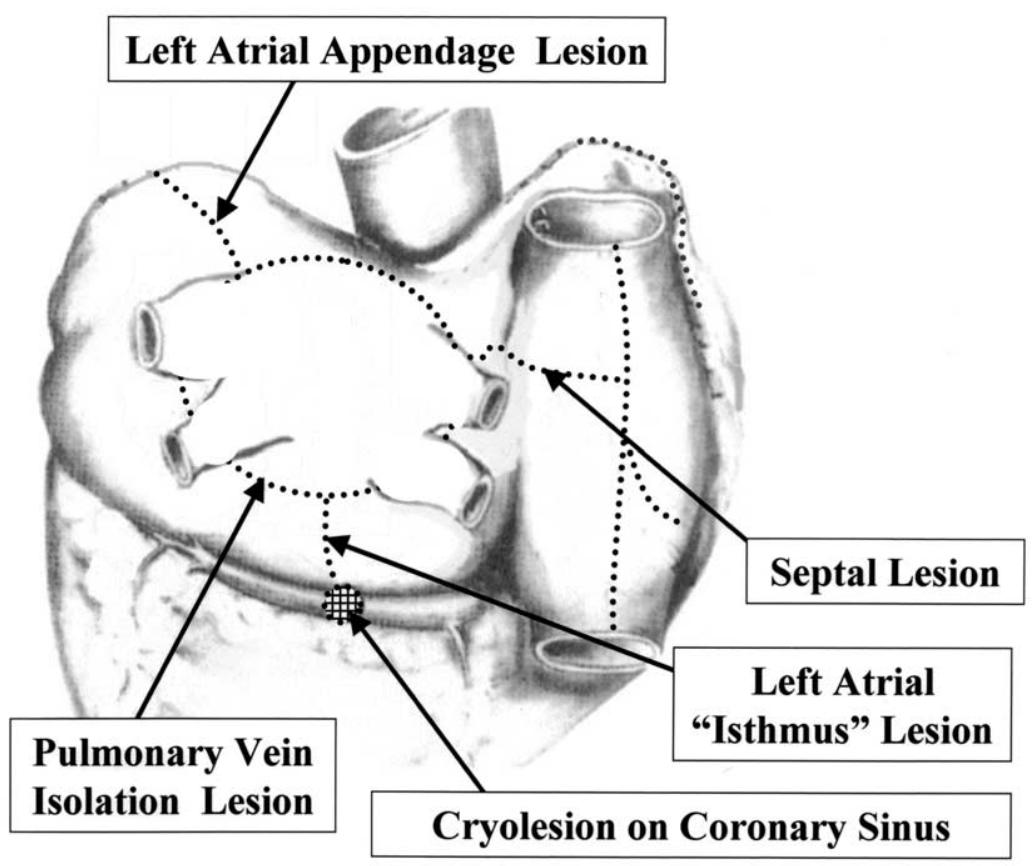

Figure 6. The 5 left atrial lesions of the standard maze III surgical procedure for atrial fibrillation.

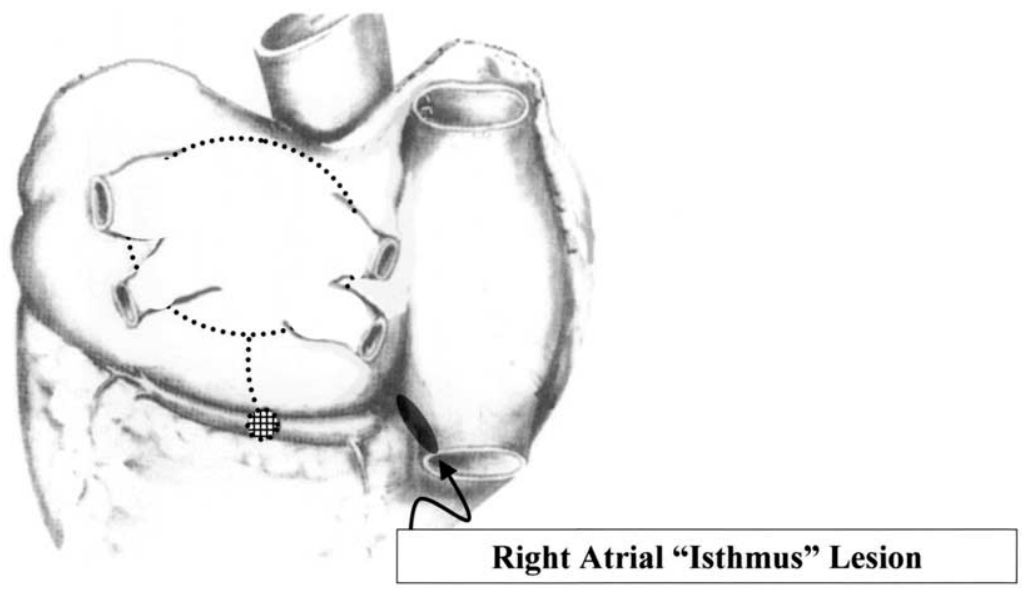

Figure 7. The mini-maze procedure for atrial fibrillation.

isthmus lesion. We call this pattern of atrial lesions the mini-maze procedure (Figure 7).

In addition to establishing this lesion pattern as the logical streamlined descendent of the original maze procedure, several years of clinical experience and enormous economic support from industry have also crystallized the characteristics of the ideal technique by which this lesion pattern should be applied. First, the procedure should be epicardial because of the inherent safety of directing the energy from the epicardium to the endocardium as opposed to delivering it from the endocardium, where overpenetration can result in catastrophic complications. Second, the energy source should be capable of penetrating epicardial fat, as well as atrial muscle, to preclude the necessity for surgical dissection of the fat before applying the energy. Third, the procedure should be capable of ablating intermittent atrial fibrillation, continuous atrial fibrillation, and atrial flutter. Fourth, cardiopulmonary bypass must be avoided. Fifth, the procedure should be amenable to endoscopic techniques or at least to truly minimally invasive techniques. Sixth, the procedure should take less than 1 hour to perform. Seventh, in the absence of complications, patients should be capable of being discharged from the hospital on the first postoperative day if not sooner. In summary, the 


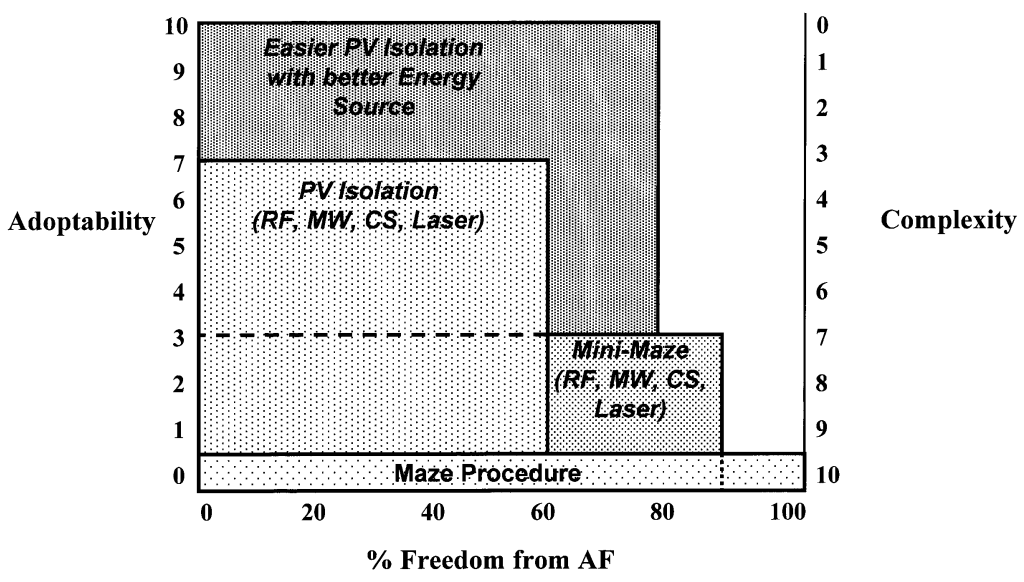

Figure 8. The relative effect on the treatment of atrial fibrillation that would occur by having an improved energy source that could be used to isolate the pulmonary veins reproducibly from the epicardial surface without cardiopulmonary bypass through a minimally invasive incision (or endoscopically) in less than an hour. $P V$, Pulmonary vein; $R F$, radiofrequency; $M W$, microwave; $C S$, cryosurgery; $A F$, atrial fibrillation.

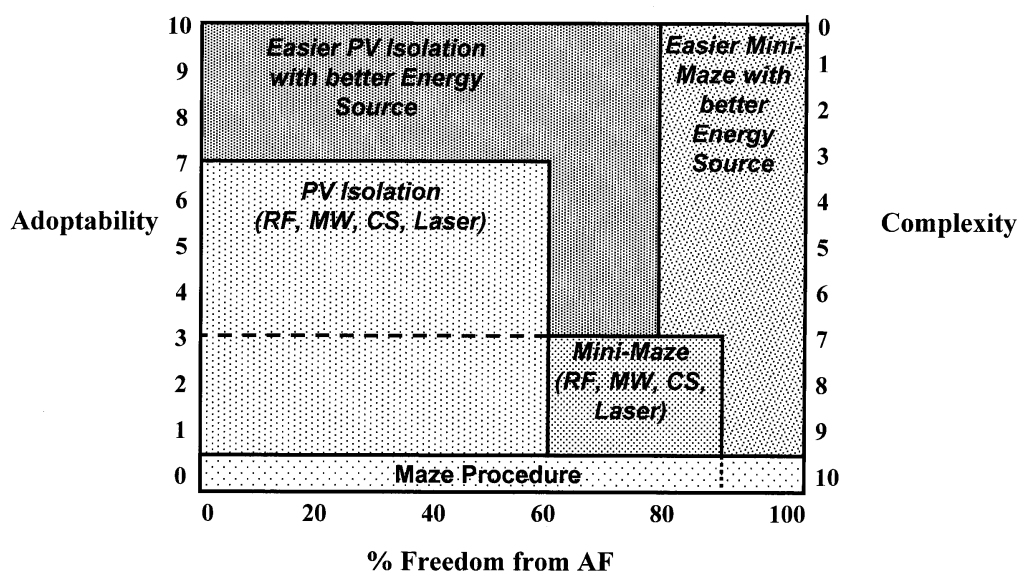

Figure 9. If the left atrial isthmus lesion could be placed from the epicardial surface by using the new energy source, thereby permitting a fully epicardial mini-maze procedure, the ideal operation for atrial fibrillation might be realized. $P V$, Pulmonary vein; $R F$, radiofrequency; $M W$, microwave; $C S$, cryosurgery; $A F$, atrial fibrillation.

ideal atrial fibrillation procedure would be performed through a minimally invasive incision (or endoscopically or robotically), off bypass, in less than 1 hour, and with hospital discharge planned for the next morning.

This ideal atrial fibrillation procedure will require the development of new energy sources that can ablate tissue in critical areas, such as the left atrial isthmus, more rapidly, uniformly, and safely than any of the energy sources now available. Assuming the development of such energy sources, pulmonary vein isolation for intermittent atrial fibrillation will immediately become more effective because of its uniform transmurality, and because of its simplicity, it would likely be adopted by virtually all surgeons (Figure 8).
The mini-maze procedure, if equally simple and effective, would then complete the picture of the ideal operation for atrial fibrillation (Figure 9). Thereafter, the number of patients cured of atrial fibrillation would no longer depend on the safety and efficacy of the surgical procedure but rather on the number of patients referred for the operation.

A final cautionary note seems warranted. Despite the promise of developing a simpler operation for atrial fibrillation that will be feasible for use in virtually all patients, it is important to remember that many of the more recent concepts, particularly those relating to the induction of atrial fibrillation, are based either on limited scientific data, on very few clinical cases, and/or on observations that were 
made in highly selected groups of patients. The electrophysiology of atrial fibrillation is unforgiving, and despite our fondest wishes, the electrophysiologic truths regarding atrial fibrillation and its treatment cannot be altered to fit our preconceived notions. It is predictable that there will always be a significant number of patients with atrial fibrillation who require the more extensive and complex surgical procedures. Nevertheless, these newer streamlined surgical approaches promise the possibility of our curing patients in the future who are currently doomed to live with their disease. Thus it behooves all of us as cardiac surgeons to take the time to learn the fundamental principles of atrial fibrillation and the rules of arrhythmia surgery that have evolved over the past 35 years. Our professional futures might well depend on it.

\section{References}

1. Harada A, Konishi T, Fukata M, Higuchi K, Sugimoto T, Sasaki K. Intraoperative map guided operation for atrial fibrillation due to mitral valve disease. Ann Thorac Surg. 2000;69(2):446-50.

2. Cox JL, Ad N. The importance of cryoablation of the coronary sinus during the Maze procedure. Semin Thorac Cardiovasc Surg. 2000;12:20-4.

3. Lammers WJ, Schalij MJ, Kirchhof CJ, Allessie MA. Quantification of spatial inhomogeneity in conduction and initiation of reentrant atrial arrhythmias. Am J Physiol. 1990;259(4 Pt 2):H1254-63.

4. Cox JL, Boineau JP, Schuessler RB, Kater KM, Lappas DG. Five-year experience with the maze procedure for atrial fibrillation. Ann Thorac Surg. 1994;56:814-24.

5. Nakagawa H, Lazzara R, Khastgir T, Beckman KJ, McClelland JH, Imai $\mathrm{S}$, et al. Role of the tricuspid annulus and the Eustachian valve/ ridge on atrial flutter. Relevance to catheter ablation of the septal isthmus and a new technique for rapid identification of ablation success. Circulation. 1996;94(3):407-24.

\section{ON THE MOVE?}

Don't miss a single issue of the journal! To ensure prompt service when you change your address, please photocopy and complete the form below.

Please send your change of address notification at least six weeks before your move to ensure continued service. We regret we cannot guarantee replacement of issues missed due to late notification.

\section{JOURNAL TITLE:}

Fill in the title of the journal here.

\section{OLD ADDRESS:}

Affix the address label from a recent issue of the journal here.

\section{NEW ADDRESS:}

Clearly print your new address here.

Name

Address

City/State/ZIP
COPY AND MAIL THIS FORM TO:

Mosby

Subscription Customer Service

6277 Sea Harbor Dr

Orlando, FL 32887
OR FAX TO:

407-363-9661

N/M Mosby
OR PHONE:

800-654-2452

Outside the U.S., call

407-345-4000 